\title{
COMPORTAMENTO CORROSIVO DE LIGAS DE MAGNÉSIO ZK30 E ZK60 EM SBF KOKUBO*
}

Tobias Bertoldi Agostini ${ }^{1}$ JulianaGrolli Lucca ${ }^{2}$ Joel da Silva Rodrigues ${ }^{3}$ Célia de Fraga Malfatti

\begin{abstract}
Resumo
O estudo de biomateriais degradáveis é um dos tópicos de pesquisa mais interessantes dos biomateriais nos dias atuais. Magnésio e suas ligas estão sendo estudados para serem usados como materiais de implantes bioabsorvíveis, para uso em dispositivos cardiovasculares ou ortopédicos. A dificuldade que se encontra em utilizar implantes à base de magnésio é a sua alta taxa de corrosão. Este trabalho tem por objetivo estudar o comportamento corrosivo de ligas de magnésio bioabsorvíveis (ZK30 e ZK60) em eletrólito que simula o fluido corpóreo (SBF Kokubo) através de técnicas de perda de massa por imersão e curvas de polarização. Os resultados indicaram uma menor resistência à corrosão da liga ZK60 no eletrólito analisado comparada com a liga ZK30.
\end{abstract}

Palavras-chave: Magnésio; Bioabsorvível; Corrosão; Biomaterial.

\section{CORROSIVE BEHAVIOR OF MAGNESIUM ALLOYS ZK30 AND ZK60 IN SBF KOKUBO}

\begin{abstract}
The study of biodegradable biomaterials is one of the most interesting research topics in biomaterials today. Magnesium and its alloys are being studied for use as bioabsorbable implant materials for use in cardiovascular or orthopedic devices. The difficulty in using magnesium-based implants is their high rate of corrosion. This work aims to study the corrosive behavior of bioabsorbable magnesium alloys (ZK30 and ZK60) in electrolyte that simulates body fluid (SBF Kokubo) through immersion mass loss techniques and polarization curves. The results indicated a lower corrosion resistance of the ZK60 alloy in the analyzed electrolyte compared to the ZK30 alloy.
\end{abstract}

Keywords: Magnesium; Bioabsorbable; Corrosion; Biomaterial.

1 Graduando de Engenharia Metalúrgica, bolsista de IC, Departamento de Metalurgia, Universidade Federal do Rio Grande do Sul - UFRGS, Porto Alegre, RS, Brasil.

2 Graduando de Engenharia Metalúrgica, Departamento de Metalurgia, Universidade Federal do Rio Grande do Sul - UFRGS, Porto Alegre, RS, Brasil.

3 Engenheiro Metalúrgico,Mestre, Doutorando, Programa de Pós Graduação em Engenharia de Minas, Metalúrgica e Materiais, Universidade Federal do Rio Grande do Sul - UFRGS, Porto Alegre, RS, Brasil.Email: joelrodrigues@charqueadas.ifsul.edu.br

4 Engenheira Metalúrgica,Doutora, Professora,Departamento de Metalurgia, Universidade Federal do Rio Grande do Sul - UFRGS, Porto Alegre, RS, Brasil. Email: Célia.malfatti@ufrgs.br. 


\section{INTRODUÇÃO}

Os biomateriais degradáveis ou bioabsorvíveis constituem uma nova classe de biomateriais consideravelmente bioativos que se espera que suportem 0 processo de cicatrização de um tecido ou órgão doente e que lentamente se degradem depois disso.

O estudo de biomateriais degradáveis é um dos tópicos de pesquisa mais interessantes dos biomateriais nos dias atuais. Os metais biodegradáveis devem degradar-se no complexo ambiente fisiológico do corpo humano com a cinética de degradação correspondente ao período de cicatrização. Os produtos de degradação devem ser transportados e eliminados do corpo e não causar reações no local ou no sistema. ${ }^{1}$

Dentre as ligas de $\mathrm{Mg}$ estudadas para aplicação biomédica destacam-se as ligas AZ91, AM50, AZ31, ZK60, WE30 entre outras. As ligas ZK30 e ZK60 se destacam pelas suas propriedades mecânicas, e sua resistência contra a corrosão[2], pela ausência do elemento alumínio, de terras raras, entre outros elementos conhecidos como nocivos ao ser humano.

A dificuldade que se encontra em utilizar implantes à base de magnésio é a sua alta taxa de corrosão. Durante a corrosão do magnésio ocorre a reação catódica de formação do gás hidrogênio, o qual pode se acumular em regiões adjacentes ao implante, podendo interferir na cicatrização dos tecidos.

Magnésio e suas ligas estão sendo estudados para serem usados como materiais de implantes bioabsorvíveis, para uso em dispositivos cardiovasculares ou ortopédicos. O conceito de usar um material metálico facilmente corrosivo para implantes não-permanente é aparentemente simples, pois é baseado em um processo de degradação espontânea. Contudo, os detalhes do processo de corrosão são muito complexos. ${ }^{3}$

O presente trabalho tem por objetivo estudar o comportamento corrosivo de ligas de magnésio bioabsorvíveis (ZK30 e ZK60) em eletrólito que simula o fluido corpóreo: SBF Kokubo. Para realizar este estudo foram utilizadas as técnicas de MEV/EDS, perda de massa por imersão e polarização potenciodinâmica.

\section{MATERIAIS E MÉTODOS}

Para o presente estudo, foram utilizadas as ligas de Magnésio ZK30 e ZK60 fornecidas pelo Departamento de Engenharia Biomecânica da Universidade Tecnológica de Delft, Holanda. As amostras foram recebidas em tarugos cilíndricos com aproximadamente $200 \mathrm{~mm}$ de comprimento e $48 \mathrm{~mm}$ de diâmetro. As amostras não passaram por nenhum tipo de tratamento térmico e a sua condição é bruta de fusão. Os tarugos foram cortados em discos com $3 \mathrm{~mm}$ de espessura e após cortados nos tamanhos necessários para as análises.

As amostras foram lixadas com lixas de carbeto de silício e polidas com pasta de diamante de $1 \mu \mathrm{m}$. Foram feitas análises de fluorescência de raios $X$, microscopia ótica e eletrônica com sonda EDS para verificação da microestrutura, perda de massa por imersão e evolução de hidrogênio.

A composição química das amostras foram obtidas através de análise química por fluorescência de raios $X$. A análise da microestrutura foi feita microscopia eletrônica de varredura com sonda EDS. 
Para o ensaio de perda de massa as amostras foram imersas em um becker com $400 \mathrm{ml}$ da solução. As amostras foram limpas em banho ultrassônico com acetona, foram medidas e pesadas em balança analítica e após isto foram imersas em solução SBF Kokubo ${ }^{4}$. O pH do eletrólito foi ajustados a 7,4 e a temperatura da estufa ajustada em $36,5^{\circ} \mathrm{C} \pm 1^{\circ} \mathrm{C}$. Após o tempo de cada ensaio as amostras foram retiradas, limpas com solução contendo $200 \mathrm{~g} / \mathrm{l}$ de $\mathrm{CrO}_{3}+10 \mathrm{~g} / \mathrm{lggNO}$ para retirada dos óxidos da superfície, lavadas com água deionizada, secas com jato de ar frio e pesadas novamente. O ph final de cada solução foi medido sendo que a cada 48horas a solução foi renovada.

Para a análise por polarização potenciodinâmica, as amostras, após processo de preparação da superfície, foram submetidas aos ensaios de polarização com intervalo de varredura de -100 à $+800 \mathrm{mV}$ em relação ao OCP, Velocidade de varredura de $1 \mathrm{mV} / \mathrm{s}$, temperatura do eletrólito de $36,5^{\circ} \mathrm{C} \pm 1^{\circ} \mathrm{C}$. O volume da célula foi de $150 \mathrm{ml}$ e o eletrodo de referência utilizado foi o de $\mathrm{Ag} / \mathrm{AgCl}$ saturado.

\section{RESULTADOS E DISCUSSÃO}

\subsection{Análise da composição química}

A Tabela 1 apresenta a composição química das amostras ZK30 e ZK60 obtidas em equipamento de fluorescência de raios $X$, da marca Thermo Scientific, modelo Niton XL3t. A composição química está de acordo com a norma ASTM B107.

Tabela 1: análise química das amostras ZK30 e ZK60 por Fluorescência de raios X.

\begin{tabular}{|c|c|c|c|c|c|c|c|c|c|c|c|c|c|c|}
\hline Amostra & Zr & $\begin{array}{c}\text { Zr } \\
\text { Error }\end{array}$ & Pb & $\begin{array}{c}\text { Pb } \\
\text { Error }\end{array}$ & Zn & $\begin{array}{c}\text { Zn } \\
\text { Error }\end{array}$ & Cr & $\begin{array}{c}\text { Cr } \\
\text { Error }\end{array}$ & $\mathbf{V}$ & $\begin{array}{c}\mathbf{V} \\
\text { Error }\end{array}$ & $\mathbf{T i}$ & $\begin{array}{c}\text { Ti } \\
\text { Error }\end{array}$ & Mg & $\begin{array}{c}\text { Mg } \\
\text { Error }\end{array}$ \\
\hline ZK30 & 0,36 & 0,01 & 0,002 & 0,001 & 3,59 & 0,07 & 0,009 & 0,003 & 0,04 & 0,01 & 0,02 & 0,01 & 95,97 & bal. \\
\hline ZK60 & 0,41 & 0,01 & 0,004 & 0,001 & 6,28 & 0,13 & 0,012 & 0,003 & 0,05 & 0,01 & 0,012 & 0,003 & 93,17 & bal. \\
\hline
\end{tabular}

\subsection{Análise por MEV/EDS}

A análise por MEV/EDS foi feita em Microscópio eletrônico de Varredura da Marca Zeiss EVO MA10, microscópio de varredura convencional de filamento de tungstênio com tensão de operação de $10 \mathrm{kV}$.

A Figura 1 apresenta a superfícies das amostras ZK30 (a) e ZK60 (b). É possível verificar intermetálicos no contorno de grão da amostra ZK60. Foi feito uma análise dos elementos presentes no intermetálico da amostra ZK60 (em destaque) e indicou a presença de zinco e magnésio neste pontos, indicando a formação no contorno de grão de um intermetálico Zn-Mg. 


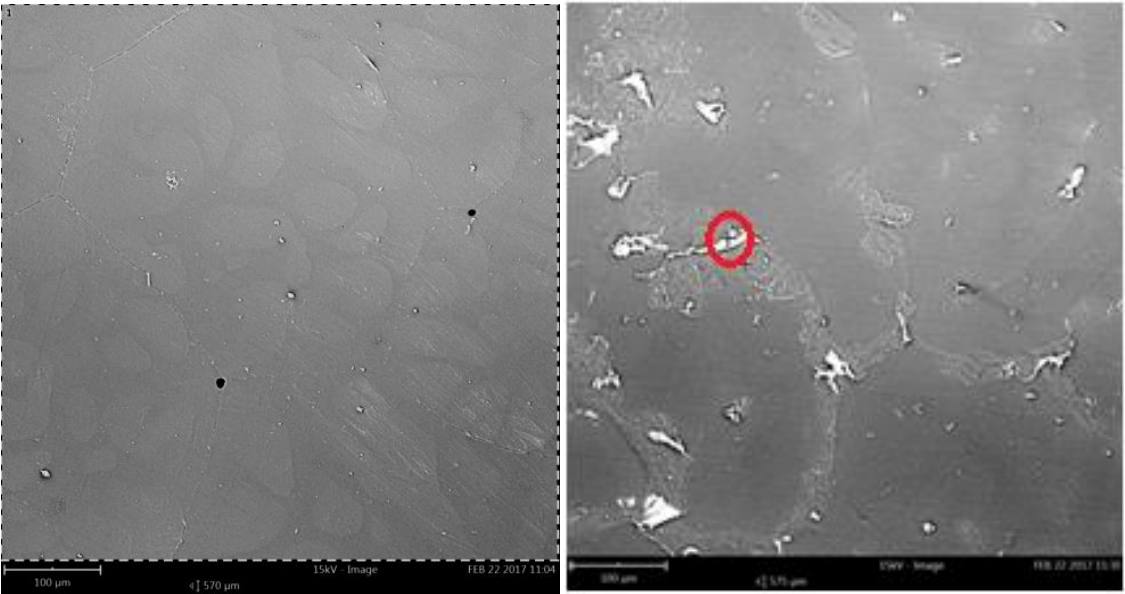

Figura 1: imagem em MEV das amostras (a) ZK30 e(b) ZK60.

\subsection{Análise de perda de massa por imersão}

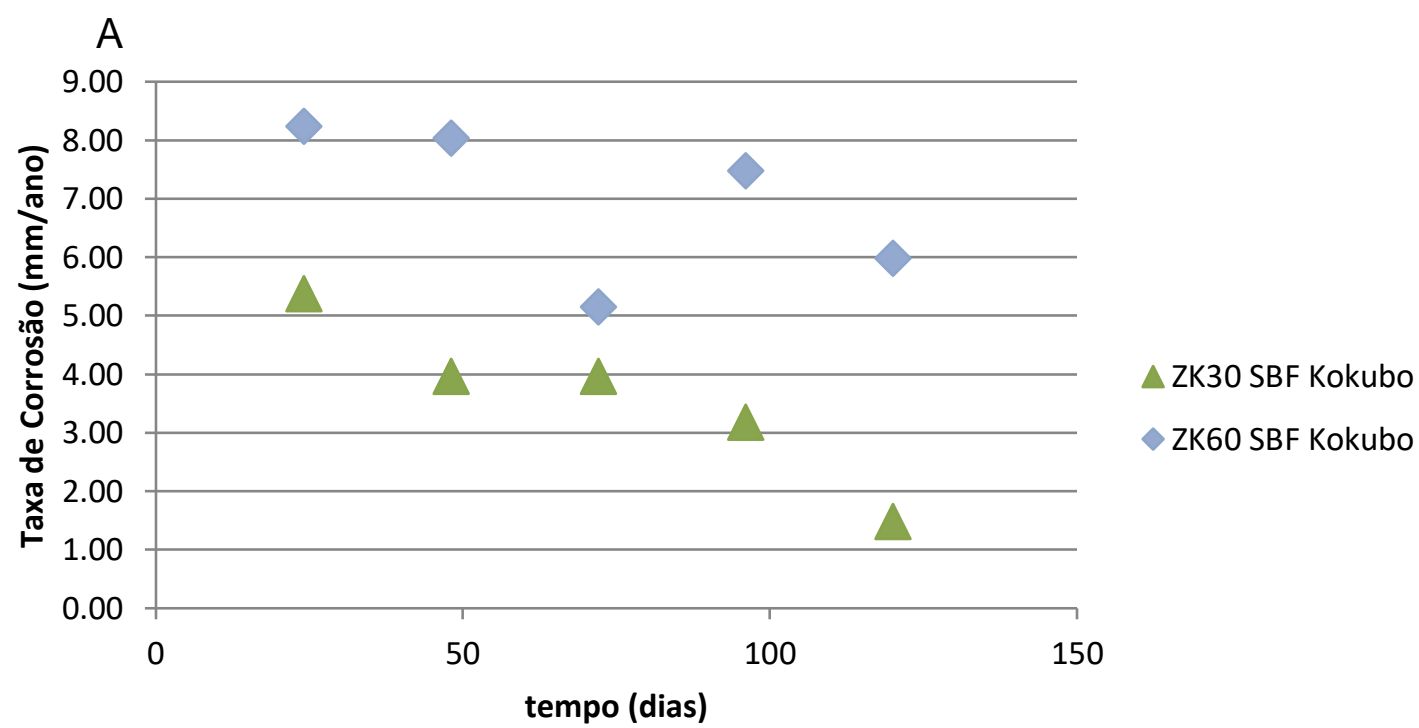

Figura 2 apresenta os valores de perda de massa por área da amostra verificada ao longo de 120 horas de análise. Percebe-se que a amostra ZK60 imersa possui a maior perda de massa por área indicando uma menor resistência a corrosão desta liga neste eletrólito analisado. 


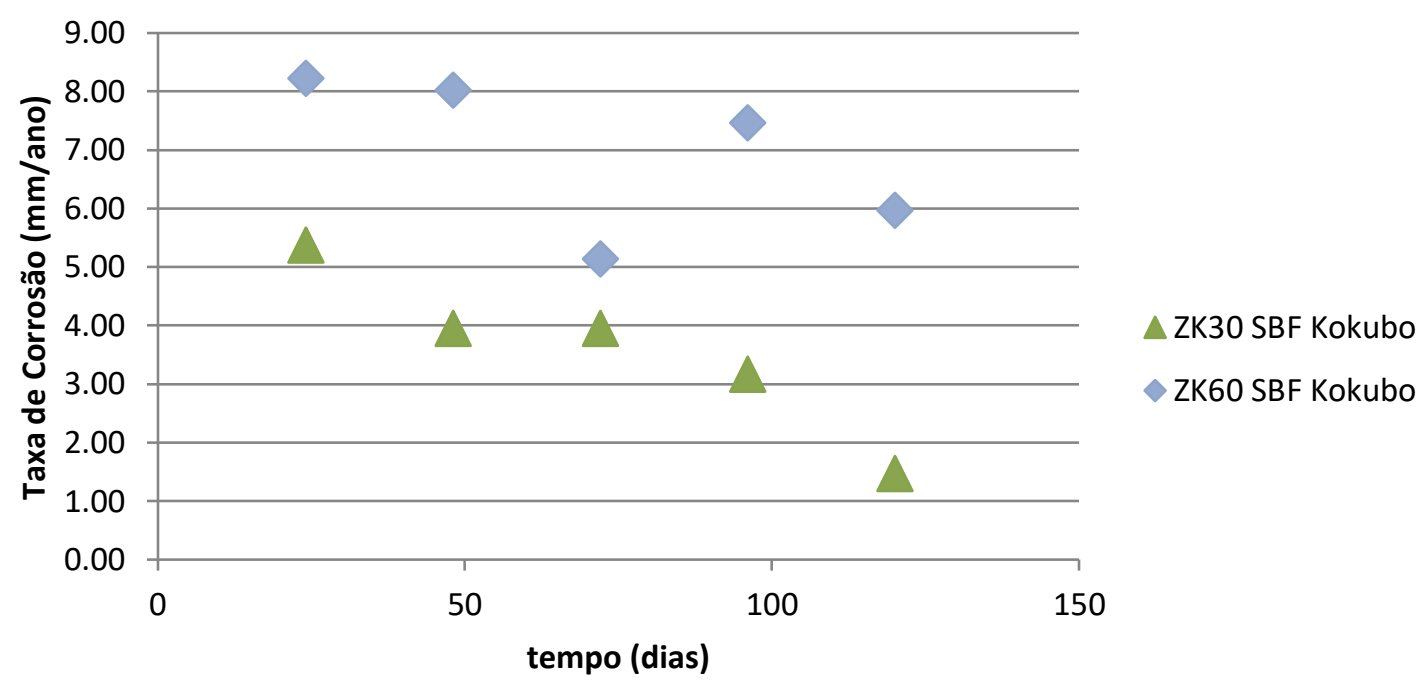

Figura 2: resultados das análises de perda de massa por imersão das amostas ZK30 e ZK60 em solução Kokubo.

A variação do $\mathrm{pH}$ com o tempo é apresentado na Figura 3. O eletrólito SBF Kokubo contendo a amostra ZK30 apresentou a menor variação de pH. Já a amostra ZK60 imersa nesta solução foi a que apresentou uma maior variação do pH no tempo analisado.

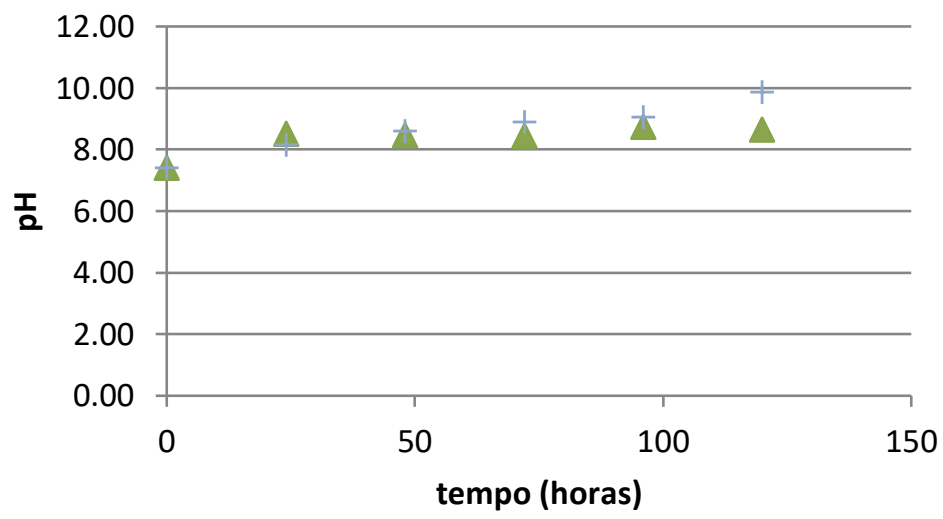

$\triangle$ ZK30 SBF Kokubo

+ ZK60 SBF Kokubo

Figura 3: variação do pH com o tempo de imersão das amostras ZK30 e ZK60.

\subsection{Análise do comportamento eletroquímico por curva de polarização}

A Figura 4 apresenta as curvas de potencial das amostras ZK30 e ZK60 em eletrólito SBF Kokubo. A amostra ZK60 apresentou um potencial de circuito aberto ligeiramente menor, em torno de $60 \mathrm{mV}$, do que a amostra ZK30, no eletrólito analisado. Ambas as curvas apresentam uma corrente limite a partir de $-1,2 \mathrm{~V}$. Esta corrente pode ser limitada pela formação de óxido sobre a superfície ou pela diminuição brusca da difusão dos íons na solução, próxima a superfície da amostra. 


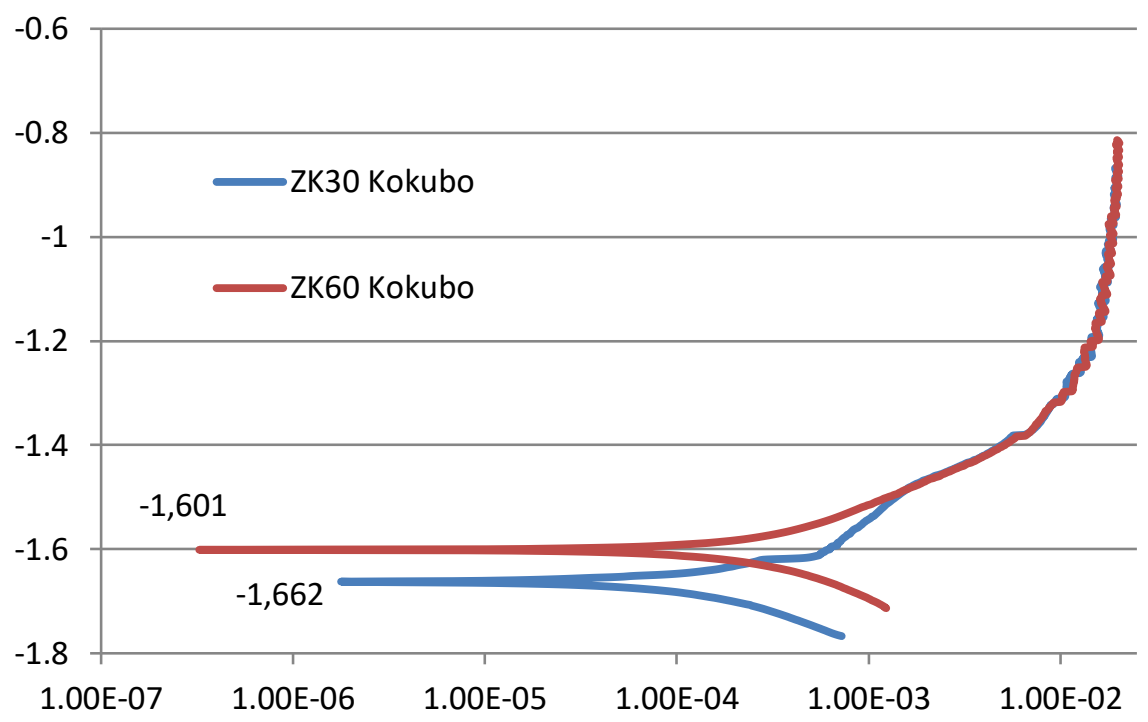

Figura 4: curvas de potencial das amostras ZK30 e Zk60 em solução SBF Kokubo.

A Tabela 2 apresenta as taxas de corrosão para as amostras ZK30 e ZK60, respectivamente, calculadas pelo software NOVA (versão 1.1). Apesar da amostra ZK30 apresentar um potencial mais negativo, a taxa de corrosão para esta amostra foi levemente menor que a taxa de corrosão da amostra ZK60.

Tabela 2: taxa de corrosão da amostra ZK30 em SBF Kokubo com respectivos dados retirados das curvas de potenciometria.

\begin{tabular}{|c|c|c|c|c|}
\hline Eletrólito & Ecorr (V) & icorr (A) & $\begin{array}{c}\text { Corrosion rate } \\
(\mathrm{mm} / \text { year })\end{array}$ & $\begin{array}{c}\text { Desvio padrão } \\
\text { da taxa de } \\
\text { corrosão }\end{array}$ \\
\hline ZK30 Kokubo & $-1,68$ & $21,6 \mathrm{E}-06$ & 3,7 & $\pm 0,2$ \\
\hline ZK60 Kokubo & $-1,61$ & $16,1 \mathrm{E}-06$ & 4,00 & $\pm 0,1$ \\
\hline
\end{tabular}

\section{CONCLUSÃO}

A composição das ligas ZK30 e ZK60 estão de acordo com o esperado para estas ligas, bem como, por ser uma liga bruta de fusão possui intermetálicos Mg-Zn nos contornos de grão conforme verificado através de MEV/EDS.

Para o ensaio de imersão e verificação da perda de massa, a amostra ZK60 apresentou uma maior perda de massa por área indicando uma menor resistência à corrosão desta liga no eletrólito analisado.

No ensaio de polarização a amostra ZK30 apresentou um potencial um pouco mais negativo que a amostra ZK60, porém, apresentou levemente uma menor taxa de corrosão que a ZK60. Isto indica uma melhor resistência à corrosão da amostra ZK30 em relação à ZK60, no eletrólito analisado. 


\section{Agradecimentos}

Agradecemos a CAPES, o CNPQ e a UFRGS pelo apoio.

\section{REFERÊNCIAS}

1 Hendra Hermawan. Biodegradable Metals: from concept to applications. New York. Springer. 2012.

2 Cheng, Ying-liang, et al. "Comparison of corrosion behaviors of AZ31, AZ91, AM60 and ZK60 magnesium alloys." Transactions of Nonferrous Metals Society of China 19.3 (2009): 517-524.

3 Sannakaisa Virtanen. Biodegradable $\mathrm{Mg}$ and $\mathrm{Mg}$ alloys: Corrosion and biocompatibility. Materials Science and Engineering B 176 (2011) 1600- 1608.

4 Tadashi Kokubo, Hiroaki Takama . "How useful is SBF in predicting in vivo bone bioactivity?". biomaterials. Volume 27. Issue 15. May 2006.pag. 2907-2915. 